\title{
Éditorial
}

\section{Quelles perspectives d'innovations en agriculture ?}

\section{Marion Guillou}

Présidente directrice générale, Institut national de la recherche agronomique (Inra),

147, rue de l'université

75338 Paris cedex 07

$<$ marion.guillou@paris.inra.fr>
\ ampleur des défis auxquels l'agriculture devra faire face dans les prochaines décennies, l'évolution rapide et la puissance même des biotechnologies, la diversité de leurs applications et de leurs effets avérés ou potentiels, et les débats que certaines d'entre elles suscitent, imposent à un organisme de recherche de ne pas rester les bras croisés.

Les biotechnologies ont renouvelé tant les outils d'investigation que les possibilités d'innovation dans le domaine du vivant. Elles constituent aujourd'hui un outil, aussi indispensable que commun, des laboratoires de biologie et génétique végétale pour leur activité de recherches sur le fonctionnement des plantes et des interactions plante-environnement.

Elles sont aussi porteuses d'innovations variées, potentiellement utiles pour relever les défis actuels et futurs de l'agriculture, de la santé ou de l'environnement. Les recherches doivent en aborder toutes les facettes pour faire en sorte que leur développement soit maitrisé et contribue à la diversification, la durabilité et la compétitivité de l'agriculture.

En France, $44 \%$ des entreprises de biotechnologies travaillent pour l'agriculture, l'agroalimentaire et la santé animale; ainsi, la France est le pays européen qui possède la plus forte proportion d'entreprises de "biotechnologies vertes". Cependant, si l'emploi dans les entreprises de biotechnologies se maintient, la proportion des "biotechnologies vertes" a baissé. Dans le même temps, la demande mondiale est en forte croissance, notamment dans les pays émergents.

\section{Un contexte}

de recherche

nécessairement

pluridisciplinaire, des voies

\section{d'innovations multiples}

Les recherches sur les biotechnologies végétales s'appuient désormais sur la connaissance des génomes et de leur expression. La France s'est dotée d'un centre national de ressources génomiques végétales ouvert sur tous les partenariats internationaux. Plusieurs génomes complets de plantes ont été séquencés: l'Institut national de la recherche agronomique (Inra) a participé à ces initiatives et en a parfois été à l'origine (vigne). D'autres projets sont en cours de réalisation, avec une forte participation française (blé, tomate, luzerne).

En sélection végétale, si l'on dispose d'une variabilité naturelle dans l'espèce, cela permet de sélectionner les meilleurs individus pour des caractères et un environnement donnés. C'est pourquoi il est primordial de conserver, gérer, caractériser et diffuser la diversité génétique, naturelle ou induite, des plantes cultivées.

L'objectif du sélectionneur de semences est en effet de rassembler dans une plante d'une espèce donnée, le maximum de caractères favorables : aptitude à la production (rendement, tolérance aux stress climatiques, résistances aux maladies et aux ravageurs) et qualité du produit. 
Jusqu'aux années 1960, le sélectionneur ne disposait que d'une seule technique : la reproduction sexuée, puis le tri a posteriori des descendants intéressants. Le processus était donc long et limité par les caractéristiques de la reproduction sexuée.

Des solutions biotechnologiques permettent progressivement de contourner ces limites :

- sauvetage d'embryons interspécifiques, régénération de plantes à partir de graines de pollen, fusion de protoplastes, qui permet le croisement entre deux espèces éloignées, quand le sauvetage d'embryons n'est pas suffisant ;

- transgenèse, qui vise à introduire dans un organisme un (ou plusieurs) gène(s) de n'importe quel autre organisme, de la même espèce ou d'une autre espèce ;

- "tilling" (Targeting Induced Local Lesion IN Genomes) qui permet de savoir si un gène auquel on s'intéresse a été muté lors d'expériences de mutation aléatoire, ou "eco-tilling" qui identifie les gènes recherchés dans une collection de plantes issues de la biodiversité existante ; - enfin, sélection assistée par marqueurs qui permet de contrôler les croisements ; en effet les marqueurs sont des fragments d'ADN, faciles à récupérer, situés à proximité des gènes d'intérêt et qui coségrègent avec eux au cours des croisements.

L'application du génie génétique dans le domaine des plantes soulève des questions de nature diverse : insertion dans un système de culture, rôle des acteurs industriels, propriété intellectuelle applicable à ces innovations, débat sur les effets sanitaires et environnementaux des plantes génétiquement modifiées susceptibles d'être cultivées et/ou commercialisées... en bref, une évaluation, globale puis au cas par cas, des risques et des bénéfices de ces innovations.

Les recherches relatives aux plantes génétiquement modifiées ne se réduisent donc pas aux projets de biologie végétale utilisant des organismes génétiquement modifiés (OGM) au laboratoire, ni aux essais au champ. Elles supposent de considérer, croiser et intégrer un ensemble beaucoup plus vaste de projets, de disciplines et d'approches.

\section{Les engagements de I'Inra}

Dans le contexte difficile des débats liés aux cultures de plantes génétiquement modifiées en Europe, l'Inra a choisi de rester "compétent " et de ne pas abandonner le secteur des biotechnologies végétales, tant en termes de création de connaissances nouvelles que d'expertise nationale et européenne, ou de propositions d'innovations.

Les réflexions au sein du Comité d'éthique, du Conseil scientifique et des équipes de recherche ont abouti à l'adoption, le 27 juin 2007, d'une politique en matière de biotechnologies végétales par le Conseil d'administration de l'Inra. Déclinée selon les différentes missions de l'Inra, elle précise la manière dont l'Institut conduit ses travaux dans ce domaine. Ainsi, l'Inra s'engage à :

- accroître les connaissances scientifiques nécessaires à la maîtrise des biotechnologies végétales ;

- cibler les innovations développées par ses équipes (qu'elles soient transgéniques ou non) sur des objectifs d'intérêt collectif qui répondent à des défis décisifs pour l'agriculture, l'alimentation et l'environnement; la transgenèse sera envisagée sur la base d'une évaluation comparative par rapport aux autres innovations possibles, lorsque ces autres alternatives ne seront pas satisfaisantes, et avec un accompagnement ouvert aux divers porteurs d'enjeux ;

- constituer des ressources génétiques publiques et accessibles pour faciliter l'appropriation des biotechnologies par les utilisateurs publics ou privés, nationaux, ou internationaux en liaison avec le Centre de coopération internationale en recherche agronomique pour le développement (Cirad), et conserver un patrimoine diversifié ;

- renforcer les capacités nationales d'expertise publique en conduisant des recherches sur l'évaluation des bénéfices, impacts et risques, à différentes échelles, des diverses formes d'innovation (transgéniques ou non).
Nous sommes donc favorables à la possibilité d'expérimenter en champ.

En effet, comment serions-nous capables de nous prononcer dans des enceintes internationales sur l'intérêt et les conditions des expérimentations et autorisations d'OGM sans capacité de test? Les essais promus par l'Inra sont des essais de compréhension, menés selon les principes de précaution, de transparence et de parcimonie. Nos essais sont donc peu nombreux et exigent d'avoir préalablement déterminé, en laboratoire et en serre, les effets prévisibles, les questions à explorer et les précautions à prendre.

Le principe de précaution exige ici que la recherche publique reste compétente, à travers une capacité scientifique maintenue, diversifiée et non appropriée. Le principe de précaution impose une recherche ambitieuse sur les biotechnologies vertes, notamment pour conserver une capacité de production compétitive et durable. Avec des programmes internationaux sur l'exploration de la biodiversité naturelle pour enrichir l'innovation variétale, avec la mise en place d'outils structurants (plates-formes public-privé ouvertes), avec le lancement de programmes nationaux sur les risques et bénéfices des OGM, sur la prospective pour les végétaux pour la biomasse du futur..

La demande de matière végétale pour des utilisations alimentaires ou non alimentaires (matériaux, chimie, énergie) est en croissance rapide et le demeurera pendant le $\mathrm{xxI}^{\mathrm{e}}$ siècle. Les évolutions en matière de stress climatique et de maladies des plantes nécessitent des innovations, pour produire, en respectant les ressources naturelles, dans ce contexte environnement global chahuté. C'est une des voies de l'agriculture écologiquement intensive - intensive en valorisation des potentialités des milieux, intensive en compétences des acteurs, intensive en innovations techniques et sociales.

Il est urgent de lancer dans cet esprit un programme international, collaboratif, ouvert et multidisciplinaire, de développement des biotechnologies vertes. 